\title{
Mass the Confined Movement of Energy
}

\author{
Zhenhua Mei', Shuyu Mei ${ }^{2 *}$ \\ ${ }^{1}$ College of Chemistry and Molecular Engineering, Qingdao University of Science and Technology, Qingdao, China \\ ${ }^{2}$ School of Medicine, Shandong University, Jinan, China \\ Email:*mzh62@163.com
}

How to cite this paper: Mei, Z.H. and Mei, S.Y. (2017) Mass the Confined Movement of Energy. Journal of Modern Physics, 8, 923-925.

https://doi.org/10.4236/jmp.2017.86058

Received: April 7, 2017

Accepted: May 23, 2017

Published: May 27, 2017

Copyright (c) 2017 by authors and Scientific Research Publishing Inc. This work is licensed under the Creative Commons Attribution International License (CC BY 4.0). http://creativecommons.org/licenses/by/4.0/

\section{Open Access}

\begin{abstract}
According to Einstein's mass-energy relation $\left(E=m c^{2}\right)$, and the discovery of neutrino oscillation fact, a new view of mass nature is proposed: The rest mass of a particle is not else something but just the confined movement of energy.
\end{abstract}

\section{Keywords}

Mass Nature, Origin of Mass, Energy, Higgs Mechanism

\section{Introduction}

Mass is the most dominative attributes in nature. Everything including bulk matter and micro particles has their rest mass nature except the medium particles such as photon and graviton etc. Mass as an original property of matter has been recognized by us for a long time since Newton's Era or even earlier. But now, by the developments of science and technology, many things in the world have changed thoroughly so far as to the attitude of mass. The mass is no longer regarded as the most basic character in science; instead, it is willing to be considered as a resultant one. As matter, mass can be destroyed and created by other reason. The mass problem or the mass forming mechanism has been referred by Higgs [1] et al. scientists.

\section{Reasoning}

In standard model of particle physics, Higgs mechanism is the mechanism where the mass (means rest mass here and following) comes from or creates in. Its key point is that, the Higgs field (that spread everywhere in the vacuum) spontaneous symmetry breaking. Where (the standard model) the massless particle and the broken Higgs field are allowed to interact each other; its mathematical expression, the new Lagrangian (where the mass term has been added constrainedly), can again satisfy the demand of symmetry in gauge transformation, oth-

${ }^{\star}$ Corresponding author. 
erwise, without the spontaneous symmetry breaking, the mass term isn't possible to add in Lagrangian under the symmetry requirement. Therefore, the mass term exists harmoniously in Lagrangian shows that the massless particle obtained its mass in the so called Higgs mechanism, and that is considered to be the origin of mass. Additionally, the predicted more than 62 physical particles by standard model have all been validated by direct or indirect experiments. The tremendous success convinced the physics community of the Standard Model devoutly.

Meanwhile, quite some of problems including the not considered graviton, neutrino possessing of the unpredicted mass, mass concerned unknown coupling coefficients, and the not unified field theory etc. issues are put forward against the Standard Model. However, most of the exclaimed problems are reasonable and pertinent.

From the point of view of this paper, we considered that the understanding of nature is a complex thing; we can't request a successful theory (e.g. the Standard Model) to be omnipotent. The exceptional false results just show the limitations of the theory, rather than something else. Symmetry is a good point of view to the world we look at; its advantage is also its limitation. So, we should examine the same thing from different degrees as to the origin of mass issue which focused on in this paper.

Studying the origin of mass, despite of the Higgs mechanism, three kinds of other evident experiment facts must be concerned. First of all, in nucleus spontaneous decaying or combining process the rest mass decrease, and result in other forms of energy. The physical process strictly obey the famous massenergy relation of Einstein $\left(E=m c^{2}\right)$. It showed us directly as we all known that the defective mass is transformed to energy. From the degree of origin of mass, we can say that defective mass is one of the forms of energy where combined. Secondly, the negative and positive electron particles will disappear when they combined, and result in two photons in opposite direction. In which the matter and even all over the rest mass are disappear, thereout we can say that mass itself is right the energy in a form. The third, an exciting fact is that the neutrino oscillation behavior has been proved by theory [2] [3] and experiments [4] [5] [6] [7] [8]. The costful discovery not only showed us the neutrinos possessing mass, but also something important behind it, that revealing or implying the mechanism of mass.

Neutrino oscillation means that the neutrino change its mass in propagation process, and the changed mass transfer to kinetic energy on itself, as well conversely turns back-part of kinetic energy return to mass in same particle. Mass is like the reservoir where the energy stored in.

Above all of these information and analysis convinced us that the rest mass is not else something but just the confined movement of energy.

\section{Discussion}

The statement that the mass being the confined movement of energy revealed 
the origin of mass. Comparing with the Higgs mechanism, this statement is consistent and complementary with the Higgs's. Here the "confined energy" is a good annotation with the "interaction" where the Higgs mechanism expressed. In other words, the interaction there means the actually energy here.

According to our mass view, the most basic character of matter is energy rather than the mass. A particle matter can exists with no mass, but can't with no energy. As the confined energy particle, mass is the physical quantity when in expressing the traveling kinetic energy and the interacting potential energy.

Now it seems that many of the energy are in confined state, i.e. the ordinary matters around us are that of mass possessing one, and only extremely few of them can be released or unblocked to normal energy state. Then a question arose why most of the mass energy is so steady, what is its fixing mechanism, and how can we explore it?

The recognition of mass in this paper is helpful to the researches of elementary particle physics.

\section{Conclusion}

Rest mass is no longer the most essential character of matter; it can be created or destroyed. We come to conclude that the rest mass is not else something but just the confined movement of energy. Frankly speaking, the conclusion is though a hypothesis but reasonable.

\section{References}

[1] Higgs, P.W. (1964) Physical Review Letters, 13, 508. https://doi.org/10.1103/PhysRevLett.13.508

[2] Pontecorvo, B. (1957) Journal of Experimental and Theoretical Physics, 33, 549.

[3] Pontecorvo, B. (1957) Zhurnal Eksperimentalnoi i Teoreticheskoi Fiziki, 34, 247.

[4] Eguchi, K., et al. (2003) Physical Review Letters, 90, Article ID: 021802. https://doi.org/10.1103/PhysRevLett.90.021802

[5] Ahn, M.H., et al. (2003) Physical Review Letters, 90, Article ID: 041801.

[6] Bilenky, S.M. and Petcov, S.T. (1987) Reviews of Modern Physics, 59, 671. https://doi.org/10.1103/RevModPhys.59.671

[7] Bilenky, S.M., Giunti, C. and Grimus, W. (1999) Progress in Particle and Nuclear Physics, 43, 1-86. https://doi.org/10.1016/S0146-6410(99)00092-7

[8] Aguilar, A., et al. (2001) Physical Review D, 64, Article ID: 112007. https://doi.org/10.1103/PhysRevD.64.112007 
Submit or recommend next manuscript to SCIRP and we will provide best service for you:

Accepting pre-submission inquiries through Email, Facebook, LinkedIn, Twitter, etc. A wide selection of journals (inclusive of 9 subjects, more than 200 journals)

Providing 24-hour high-quality service

User-friendly online submission system

Fair and swift peer-review system

Efficient typesetting and proofreading procedure

Display of the result of downloads and visits, as well as the number of cited articles Maximum dissemination of your research work

Submit your manuscript at: http://papersubmission.scirp.org/

Or contact jmp@scirp.org 